\title{
Profiling harmful medication errors in an acute Irish teaching hospital
}

\author{
E.C. Relihan*, S.A. Ryder†, B. Silke*
}

*St. James's Hospital, James's Street, Dublin 8, Ireland.

$\dagger$ School of Pharmacy and Pharmaceutical Sciences, Trinity College Dublin, Dublin 2, Ireland.

E-mail: erelihan@stjames.ie, sryder@tcd.ie

Note: This is an author-created version of an article accepted for publication in the Irish Journal of Medical Science following peer review.

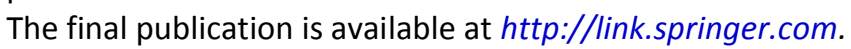

Direct links to the definitive publisher-authenticated version [Relihan EC, Ryder SA, Silke B. Profiling harmful medication errors in an acute Irish teaching hospital. Ir J Med Sci. 2012 Dec; 181(4):491-7]:

http://link.springer.com/article/10.1007\%2Fs11845-012-0804-y

http://dx.doi.org/10.1007/s11845-012-0804-y

\begin{abstract}
Background Medication error reporting systems in hospitals are faced with the challenge of processing vast numbers of reports which identify a myriad of safety issues. With such large volumes of data and limited resources it makes sense to adopt a prioritisation approach. Several published studies have focused solely on the subset of errors which cause patient harm. The majority of such research has concerned the individual analysis of criteria associated with medication errors. However, the research described here used an alternative approach which involved linking the three criteria of medication class, patient outcome, and type of error, in order to describe the medication-related scenarios presenting greatest risk to the organisation.
\end{abstract}

Aims To identify the highest-priority medication-related risks in an acute teaching hospital. To profile harmful medication errors submitted to a voluntary reporting system in a tertiary healthcare setting in Ireland.

Methods A database of medication errors, reported via an internal voluntary reporting system over a 5-year period, was analysed. The criteria of medication class, patient outcome and type of error were analysed separately and then crosstabulated.

Results The medication classes, error types and adverse patient outcomes most frequently associated with harm were identified. The cross-tabulation highlighted ten priority risk areas which accounted for the majority of patient harm.

Conclusions A cross-tabulation strategy for prioritising medication-associated risks was successfully applied to a hospital database comprising medication errors. The profile developed for harmful medication errors in this acute tertiary healthcare setting was broadly in line with that published for error reporting systems internationally.

Keywords Medication errors • Patient safety

\section{Introduction}

In the Institute of Medicine report To Err is Human, the authors expressed the view that 'given current knowledge about the magnitude of the (patient safety) problem, it would be irresponsible to expect anything less than a $50 \%$ reduction in errors over 5 years' [1]. In relation to medication safety, this goal presented an enormous challenge for healthcare, given that medication errors are associated with a variety of medications, occur in all types of patients and at all stages of the medication use process, and involve multiple contributory factors [2]. Over time, it was realised that a 50\% reduction in medication errors would only be possible by identifying and targeting the most frequently arising and severe adverse events, to ensure the most efficient use of resources [2]. Accreditation organisations advocate that individual healthcare organisations identify their top risks in relation to medications at a local level and devise tailored strategies to address these $[3,4]$.

Only a few published studies have described the separation of medication errors which resulted in adverse patient outcomes from the overall dataset and analysis of this subset of harmful events in detail [2, 5-10]. Furthermore in general, studies of medication errors focus on the individual descriptive criteria of harmful errors in isolation, and therefore lack the necessary detail to describe the nature of specific errors where resources need to be targeted. However, one US research group (Winterstein et al.) has described an effective methodology for prioritisation of medication risks in a hospital environment $[9,10]$. This involves a three-way cross-tabulation of the criteria of medication class, type of error, and adverse patient outcome, thereby enabling a majority of ostensibly unrelated data to be organised into a few distinct event types. 
We are not aware of any published application of this methodology by other researchers nor of its application to any healthcare setting outside the US.

Public hospitals in Ireland are required to submit clinical error data to a national indemnifying body — the Clinical Indemnity Scheme (CIS) - via an electronic reporting system (STARSWeb) [11]. However in relation to medication errors, the CIS database has significant limitations. First, information on the medication classes involved in errors is not collected [12, 13]. Second, the impact of medication errors is unknown in the majority of cases as patient outcome data is not specified for almost two-thirds of the events reported [13]. It is therefore not possible to use the CIS dataset to develop a profile of those medication error types which present the greatest risk to patients in Irish hospitals. Just two studies have previously been published regarding trends in medication errors in the acute hospital setting in Ireland; both of these have involved analysis of data amalgamated from a number of hospitals [14, 15]. However, neither publication encompassed a detailed analysis of the subset of medication errors which cause patient harm in this setting.

\section{Aims}

The aims of this study were:

- To apply the cross-tabulation analysis proposed by Winterstein to a database of medication errors to identify the priority risk areas where resources should be targeted.

- To develop a detailed profile of medication errors causing patient harm in an acute hospital setting in Ireland.

- To compare the profile of harmful medication errors with similar data internationally.

\section{Methodology}

This study was undertaken in an acute, teaching hospital in Ireland (Hospital X) which comprises over 1,000 inpatient and day-case beds. All major specialities, with the exception of paediatrics and obstetrics, are covered. The hospital is the national tertiary referral centre for several specialities including haematology/oncology, burns, and plastics.

A retrospective analysis was undertaken of the medication errors reported to the hospital's voluntary internal medication error reporting system between January 1, 2005, and December 31, 2009. Data mining involved applying clustering analysis based on pivot tables using Microsoft Office Excel $2000^{\circledR}$, version 9.0.

Both the complete dataset and the subset of errors which caused patient harm were analysed in relation to the criteria of error type and medication class. Medications involved in errors were categorised according to the World Health Organization (WHO) Anatomical Therapeutic Chemical (ATC) classification system [16]. Medication classes were defined for all errors that involved a single medication. Errors involving multiple medications were excluded from the analysis of medication class.

Errors were severity scored according to the National Coordinating Council Medication Error Reporting Programme (NCC MERP) system, where errors are assigned a letter from $A$ to I depending on the level of adverse impact, if any, on the patient [17]. The NCC MERP system was our preferred system as it is the one used by US researchers to date, and therefore it facilitated comparison of our results with published international trends.

Statistical analysis (Chi square analysis using Fisher's exact test where $p$ values were calculated using Monte Carlo simulation) was applied to investigate if the percentage of errors assigned to the most frequently reported medication classes and type categories for harmful errors was proportionate to what would be expected based on the overall dataset.

For the subset of medication errors which had caused patient harm, the adverse outcomes for patients were classified according to the major organ systems affected (such as cardiovascular, respiratory, endocrine) or by other critical signs or symptoms (e.g., uncontrolled pain) [10]. Cross-tabulation was then undertaken of the three criteria of error type, medication class, and patient outcome, to identify those events presenting greatest risk to the organisation.

\section{Results}

\section{Dataset}

The dataset analysed consisted of 4,074 medication errors. This comprised potential errors/near misses, i.e., MERP classes A and B (1,363, 33.4\%) and actual errors, i.e., MERP classes C-I (2,711,66.6\%). Of the actual errors, 250 (5.3\% of all events reported) resulted in harm, i.e., were classified as MERP E-I. In the vast majority $(85.6 \%)$ of these cases, any harm caused was temporary and minor.

\section{Types of error}

In the case of the overall dataset (MERP A-I) of a total of 22 categories of error type, the 7 most frequently reported accounted for $69.0 \%$ of the total number of errors: 'missed dose', 'dose incorrect', 'frequency/time incorrect', 'medication incorrect', 'documentation error', 'infusion issue', and 'duplicated dose' (Fig. 1). For the subset of harmful errors (MERP EI), the contribution of each error type was analysed to investigate if it was proportionate relative to the overall dataset. Of 
the seven most frequently reported categories, those of 'dose incorrect' and 'infusion issue' accounted for significantly disproportionately more harm, while the categories of 'frequency/time incorrect' and 'documentation error' accounted for significantly disproportionately less harm (Fisher's exact test: $\mathrm{df}=26, n=3,258, \chi^{2}$-value $=1.244 \times 10^{2}, p<0.0001$ ). 'Incorrect dose' errors, for example, accounted for just $12.2 \%$ of errors in total but they were associated with $19.2 \%$ of those errors causing harm. Conversely, although 'frequency/time incorrect' errors accounted for $12.0 \%$ of all reported errors, just $7.2 \%$ of events which caused harm were associated with this category.

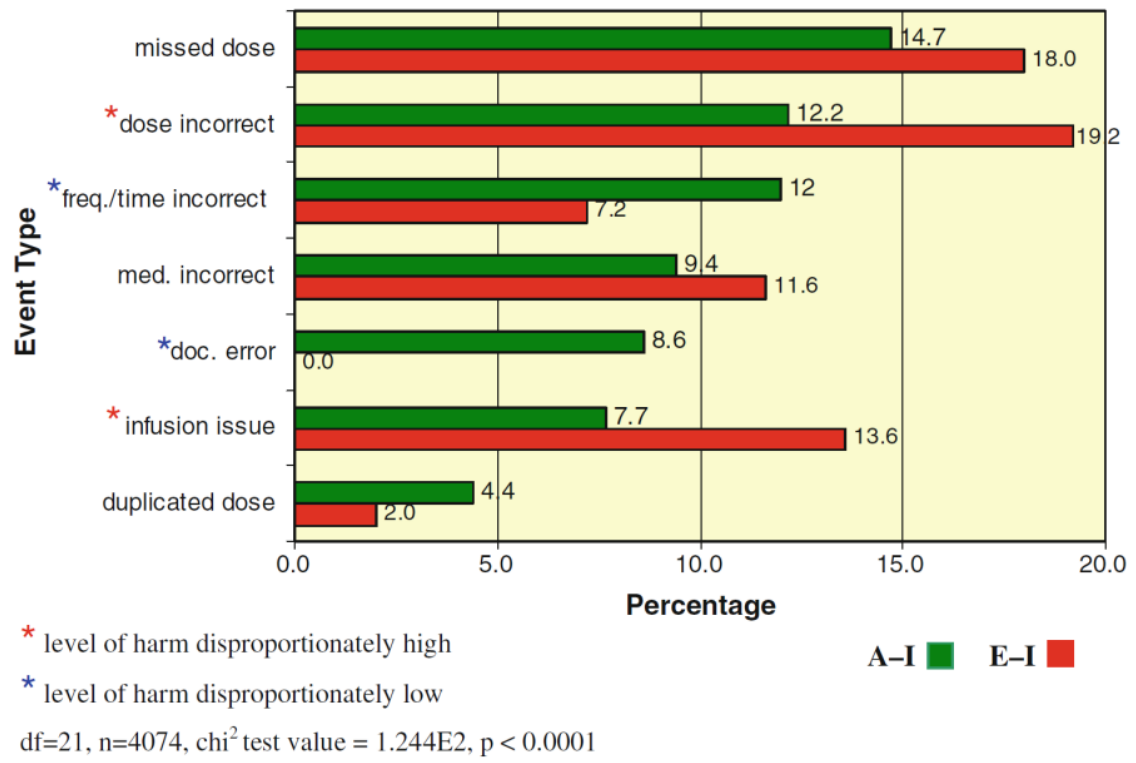

Fig. 1 Percentage of event types identified for MERP A-I $(n=4,074)$ and MERP $E-I(n=250)$ errors for the seven most frequently occurring categories

\section{Medication classes involved in errors}

The 8 most frequently reported medication classes, from a total of 27 classes, accounted for three-quarters (75.8\%) of the errors which caused harm: anti-neoplastics/immunomodulators, cardiovascular agents, anti-infectives, opiates, antithrombotics, psycholeptics/psychoanaleptics, insulin and electrolytes/fluids (Fig. 2). Of these eight classes, two classes caused significantly disproportionately more harm: opiates and insulin, and two caused significantly less: antineoplastics/immunomodulatory agents and anti-thrombotics (Fisher's exact test: $\mathrm{df}=26, n=3,528, \chi^{2}$-value $=1.05 \times 10^{2}$, $p<0.0001$ ). For example, opiates represented just $11.9 \%$ of errors overall, but were implicated in $24.5 \%$ of those errors causing harm to patients.

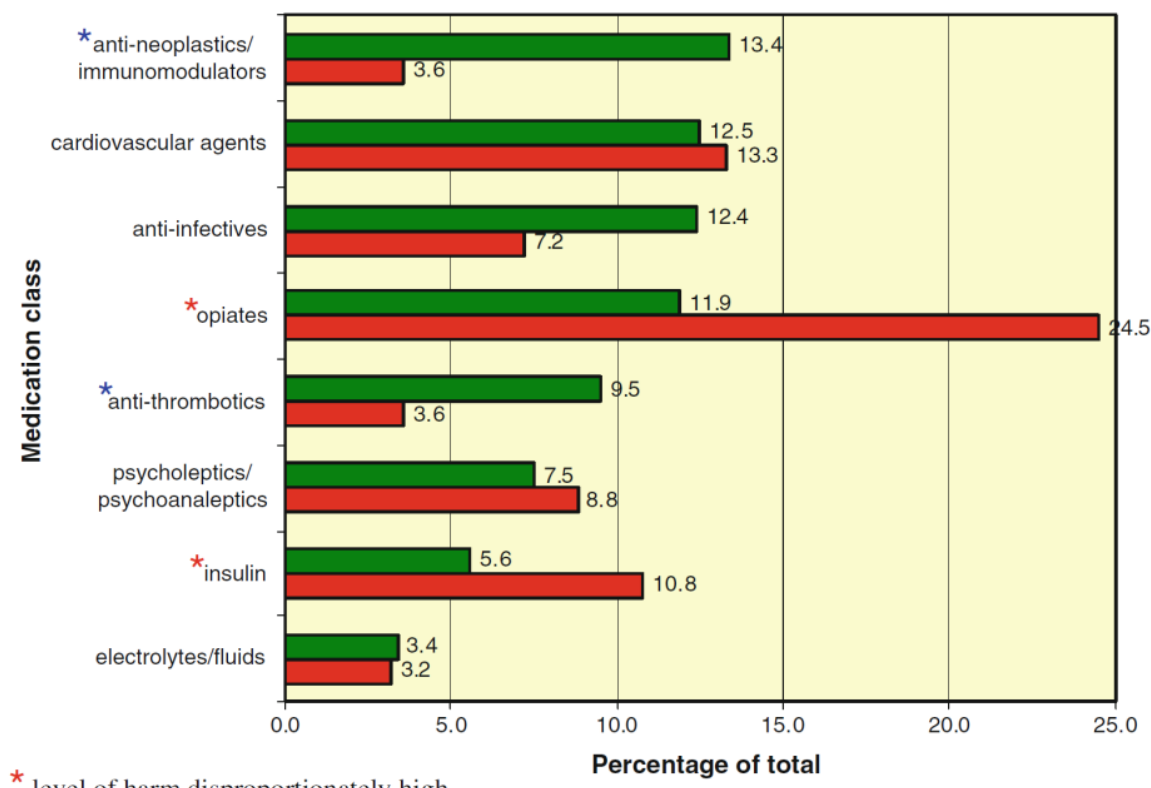

* level of harm disproportionately high

Percentage of total

* level of harm disproportionately low

$\mathrm{df}=26, \mathrm{n}=3258, \mathrm{chi}^{2}$ test value $=1.058 \mathrm{E} 2, \mathrm{p}<0.0001$

Fig. 2 Percentage of medication classes identified for MERP category A-I $(n=3,528)$ and MERP category E-I $(n=249)$ errors for the eight most frequently occurring categories 


\section{Patient outcomes}

Very distinctive trends were apparent in relation to the type of adverse impact on patients, with a majority of errors accounted for by a minority of outcome categories (Table 1). The three most frequently occurring categories cardiovascular, pain, and endocrine - accounted for over $43 \%$ of all the adverse outcomes for the harmful errors.

Table 1 Frequency of adverse patient outcomes associated with MERP category $\geq$ E errors $(n=250)$

\begin{tabular}{|c|c|c|}
\hline Outcome & No. & (\%) \\
\hline Cardiovascular: hypotension, hypertension, bradycardia, tachycardia, arrythmias & 39 & $(15.6)$ \\
\hline Pain: uncontrolled pain due to inadequate pain relief; pain resulting from insufficient dilution of intravenous medication & 39 & $(15.6)$ \\
\hline Endocrine: hypoglycaemia and hyperglycaemia & 30 & $(12.0)$ \\
\hline Neurological: seizures, rigors, exacerbation of tremor, dizziness, excessive sedation/somnolence & 30 & $(12.0)$ \\
\hline Allergic/cutaneous: rash, numbness, erythema, itch, pain/discomfort & 25 & $(10.0)$ \\
\hline Gastrointestinal: nausea, vomiting & 19 & $(7.6)$ \\
\hline Respiratory: respiratory depression, shortness of breath & 16 & $(6.4)$ \\
\hline Neuropsychiatric: confusion, agitation, aggression, light-headedness & 15 & $(6.0)$ \\
\hline Hepatic/renal/electrolytes: impaired hepatic/renal function, hypokalaemia, diuresis, dehydration & 15 & $(6.0)$ \\
\hline Haematologic: neutropenia, pancytopenia, haemorrhage, epistaxis, anaemia & 11 & (4.4) \\
\hline Increased length of stay & 6 & (2.4) \\
\hline Exacerbation of infection & 2 & (0.8) \\
\hline Musculoskeletal: dystonia, unplanned intubation & 2 & (0.8) \\
\hline Other & 1 & (0.4) \\
\hline
\end{tabular}

\section{Cross-tabulation of error type with medication class and adverse patient outcome}

When event type, medication class, and adverse outcome were cross-tabulated for the subset of errors causing harm, $70 \%$ of these errors were accounted for by just 10 event categories (Table 2).

Table 2 Ten most frequently occurring medication errors causing harm

\section{Medication safety event causing harm}

Pain associated with opiates and anaesthetics mainly resulting from missed/omitted/delayed doses, underdosing, or administration of the incorrect opiate preparation

Hypo/hypertension or arrythmias associated with cardiovascular or adrenergic/dopaminergic agents, mainly related to incorrect/missed doses, incorrect medication, or duplication of doses/therapy

Endocrine errors associated with insulin

Hypoglycaemic episodes associated predominantly with insulin overdoses

Hyperglycaemic episodes (including hyperketosis and diabetic ketoacidosis) associated with missed/omitted insulin doses

Excessive sedation, gastrointestinal upset, neuropsychiatric symptoms, or respiratory depression associated with opiates, arising mainly from overdoses, administration to the incorrect patient, or unauthorised self-administration

Neuropsychiatric and neurological reactions (agitation, aggression, confusion, somnolence) associated with psychotropics/other CNS agents, mainly as a result of missed/omitted doses or administration of the incorrect dose/medication

Allergic/cutaneous reactions associated with antibiotics as a result of infusion at the incorrect rate or concentration, or administration despite a documented history of allergy

Gastrointestinal or haematologic adverse effects associated with anti-neoplastic agents, as a result of overdoses, administration at the incorrect time, or omission of anti-emetic therapy

Seizures associated with missed or incorrect doses of anti-epileptics

Electrolyte disturbances, dehydration, and renal impairment associated with missed fluids/electrolytes and incorrect doses, or insufficient monitoring of nephrotoxic drugs

Major/minor haemorrhage associated with warfarin or enoxaparin as a result of overdoses, duplicated doses/therapy, or failure to monitor

No. (\% of total no. of

harmful errors, $n=250$ )

$35 \quad(14.0)$




\section{Discussion}

Comparison of Hospital $X$ data with published research was complicated by a lack of consistency across studies in relation to the taxonomy applied to the criteria of error type, medication classification and patient outcome. In addition, considerable variation exists in relation to the terminology used to describe harmful medication incidents. For example, some authors [8, 10] analyse 'adverse drug events (ADEs)' rather than medication errors resulting in harm. An 'ADE' may be defined as 'an injury resulting from a medical intervention relating to a drug' [8] and it therefore encompasses both adverse drug reactions and medication errors. In contrast, other studies restrict their analysis to medication errors alone, which have been defined by the NCC MERP as preventable events 'that may cause or lead to inappropriate medication use or patient harm while the medication is in the control of the health care professional, patient, or consumer' [18]. The NCC MERP definition was the one applied in this study. To address this issue of variation in terminology, the WHO has developed the International Classification of Patient Safety (ICPS) which aims to define and harmonise patient safety concepts into a single internationally agreed taxonomy [19]. The ICPS is as yet just a conceptual framework; however, the WHO is currently working to transform the framework into a true classification system. Once complete, accepted universally, and incorporated into research, it should allow for more meaningful comparison between studies in the future.

Finally, comparison of Hospital $X$ data with that from other agencies was only possible where the subset of medication errors which had caused patient harm was isolated and analysed separately from the total dataset. This factor limited the comparison of Hospital $X$ to just one [15] of the two Irish studies published in relation to medication errors, three major national reporting programmes in the UK [6, 7], US [20], and Canada [5]; and a small number of relevant small-scale studies undertaken in individual hospitals or groups of hospitals $[2,8-10]$.

\section{Types of error}

In Hospital $\mathrm{X}$, the most frequently arising error type resulting in harm was dosage errors followed by missed doses. This finding is very similar to those of other researchers. In a review of ten studies of medication errors, Kanjanarat [2] found that incorrect doses resulted in most harmful errors. Analysis of data from the UK National Reporting and Learning System (NRLS) [7] and MedMARx [20] in the US have also highlighted missed doses and incorrect doses as the most frequently reported error types causing harm.

\section{Medication classes involved in errors}

In relation to the classes of medication associated with harm, the data from national reporting systems in other countries and individual hospital studies reflect the findings for Hospital $X$, although the ranking of the classes of medications differs between them. As with Hospital X, the 2007 collaborative analysis of medication errors reported to four Irish hospitals found cardiovascular and endocrine agents to be amongst the top four classes of medications causing harm [14]. Of note is that opiates did not feature at all in the collaborative study whereas it was the class of agent highlighted as causing greatest harm in Hospital X.

Large-scale databases from other countries have consistently identified opiates as one of the classes of medications causing the greatest degree of harm. Data reported to the NRLS between January 2005 and June 2006 found that the medications most frequently associated with severe harm were opiates, anticoagulants, anaesthetics, and insulin [6]. Analysis of 2007 NRLS data highlighted cardiovascular agents as the most harmful class, followed by anti-infectives, opiates, and anticoagulants [7]. Bates [8] also found that analgesics comprised the leading class of harmful medications, with the individual opiates of morphine, meperidine and oxycodone collectively accounting for the majority of the group. Similarly the Institute for Safe Medication Practices (ISMP) has identified insulin and opiates/narcotics as the classes of medications most likely to cause harm to patients [5, 21]. In Kanjanarat's review [2], six of the ten studies assessed identified cardiovascular agents as the class most frequently associated with medication errors, followed by central nervous system (CNS) agents and analgesics (with opiates being the leading drug class in this category). Finally, two studies undertaken by Winterstein $[9,10]$ highlighted many of the same classes of agents, but the ranking of classes differed. In the first study of harmful medication errors, the medication class most commonly associated with errors was anticoagulants, followed by opiates and insulin [9], whilst the second study pinpointed anti-infectives as being of greatest risk followed by CNS agents (including opiates) [10].

\section{Patient outcomes}

Consistent with the results for the frequencies of medication classes associated with medication errors in Hospital X, cardiovascular adverse effects were the most common category of harmful outcome, followed by uncontrolled pain, and then endocrine events. Winterstein [10] has hypothesised that cases of therapeutic failure (i.e., where effective medications are either not prescribed at all or are under-dosed) are generally under-reported in voluntary reporting systems, even though they contribute substantially to ADEs. Contrary to Winterstein's hypothesis, however, the second most common category of adverse patient outcome for Hospital $\mathrm{X}$ was associated with therapeutic failure, i.e., uncontrolled pain due to inadequate pain relief. This patient outcome finding is consistent with the analysis of error type for harmful medication 
errors in Hospital $\mathrm{X}$ which identified missed doses (i.e., errors of omission) as the second most frequently occurring category.

\section{Cross-tabulation of error type with medication class and adverse patient outcome}

Despite the heterogeneity of medication errors, the cross-tabulation of the data reported to the voluntary reporting system in Hospital X revealed that a small number of error types, medication classes, and adverse patient outcomes constituted a substantial proportion of events which caused harm, i.e., just ten event types were found to account for $70 \%$ of the harmful errors. The notion of a majority of effects arising from a minority of causes (the Pareto principle) was also noted by Winterstein $[9,10]$ to be applicable to the results of this type of cross-tabulation. Furthermore, there was a substantial degree of overlap between the event types identified by our research and Winterstein's analyses. In both cases, for example, toxicity arising from opiate overdoses, and hypoglycaemic effects associated with insulin overdoses, were amongst the top five most frequently reported events [9, 10]. Likewise Kanjanarat [2] identified the most common event types to be inappropriate dosing of cardiovascular medications resulting in undesired cardiac reactions; combinations of psychoactive agents or opioids associated with over-sedation/respiratory depression; overdoses of anticoagulants; and use of anti-infectives despite history of allergy, resulting in allergic reactions. All of these event types with the exception of that relating to anti-infectives/allergy featured amongst the top ten events identified by our analysis.

A possible criticism of the three-way cross-tabulation technique we applied in this study is that although it provides a very useful overview of the harmful medication errors in an organisation, this high-level analysis does not take into account other criteria which are of value when profiling medication-associated risks and designing action plans to address them. For example, the methodology does not consider the stage of the medication-use-process involved, i.e., prescribing (on admission, during the inpatient stay, or at discharge), dispensing, administration and/or monitoring of medication. Future applications of the methodology could investigate the application of this fourth layer of analysis to medication error data to further refine this prioritisation technique.

\section{Conclusions}

We successfully applied a three-way cross-tabulation analysis of the criteria of medication class, error type and patient outcome to the medication errors reported to a voluntary reporting system in an acute hospital in Ireland. This is the first application of this methodology outside a US setting as a means of identifying institution-specific medication safety priority areas and targeting the finite resources to the initiatives most likely to reduce patient harm in the future. Application of this methodology to Hospital $X$ resulted in comparable findings to those described by Winterstein, i.e., a majority of harm was caused by a minority of event types and the same or similar combinations of medication classes, error types, and patient outcomes were highlighted as presenting the greatest risk of patient harm. Although comparison of the data in Hospital $X$ with international studies was limited by variation in the error taxonomy applied, the trends in our hospital were found to be broadly in line with the findings of voluntary reporting systems in other jurisdictions.

\section{Conflict of interest None.}

\section{References}

1. Kohn LT, Corrigan JM, Donaldson MS (eds) (1999) To err is human: Building a safer health system. Institute of Medicine, Washington DC. Available at: http://www.iom.edu/Reports/1999/To-Err-is-Human-Building-A-Safer-Health-System.aspx

2. Kanjanarat P, Winterstein A, Johns T, Hatton R, Gonzalez-Rothi R, Segal R (2003) Nature of preventable adverse events in hospitals: a literature review. Am J Health Syst Pharm 60(17):1750-1759. PMID: 14503111.

3. Rich DS (2004) New JCAHO medication management standards for 2004: changes related to high-risk medications. Standard MM.7.10 The organization develops processes for managing high-risk or high-alert medications. Am J Health Syst Pharm 61(13):1349-58. PMID: 15287230. Available at: http://www.medscape.com/viewarticle/482368_11

4. Australian Council on Healthcare Standards (ACHS) (2006) Evaluation and Quality Improvement Program (EQuIP) 4 Guide, Part 1: Accreditation, standards, guidelines. July 2006. Available at: http://www.achs.org.au/EQulP4Guide/

5. Institute for Safe Medication Practices Canada (ISMP Canada) (2006) Top 10 drugs reported as causing harm through medication error. ISMP Canada Safety Bulletin 6(1). Available at: http://www.ismp-canada.org/download/safetyBulletins/ISMPCSB200601Top10.pdf

6. NHS National Patient Safety Agency (2007) Safety in doses: Medication safety incidents in the NHS. Fourth report from the Patient Safety Observatory. National Patient Safety Agency, London. Available at: http://www.nrls.npsa.nhs.uk/resources/?Entryld45=59822

7. NHS National Patient Safety Agency (2009) Safety in doses: improving the use of medicines in the NHS. Learning from national reporting 2007. National Reporting and Learning Service. National Patient Safety Agency. Available at: http://www.nrls.npsa.nhs.uk/resources/?entryid45=61625

8. Bates D, Cullen D, Laird N (1995) Incidence of adverse drug events and potential adverse drug events; implications for prevention. $J$ Am Med Assoc 274:29-34. PMID: 7791255. DOI: 10.1001/jama.1995.03530010043033 
9. Winterstein A, Hatton R, Gonzalez-Rothi R, Johns T, Segal R (2002) Identifying clinically significant preventable adverse drug events through a hospital's database of adverse drug reaction reports. Am J Health Syst Pharm 59(18):1742-1749. PMID: 12298112

10. Winterstein A, Johns T, Rosenberg E, Hatton R, Gonzalez-Rothi R, Kanjanarat P (2004) Nature and causes of clinically significant medication errors in a tertiary care hospital. Am J Health Syst Pharm 61(18):1908-1916. PMID: 15487880

11. Clinical Indemnity Scheme. Available at: http://www.stateclaims.ie/ClinicallndemnityScheme/introduction.html

12. (2008) STARSWeb evaluation project: a report from the Health Service Executive, the Health Information and Quality Authority, and the State Claims Agency. Available at: http://www.hiqa.ie/publication/starsweb-evaluation-project

13. STARSWeb statistics 2009. Clinical Indemnity Scheme. Available at: http://www.stateclaims.ie/ClinicallndemnityScheme/starswebStats.html

14. Kirke C, Tighe P, Colohan G, Harnett B, Creaton G, Delaney T (2007) A collaborative study of medication safety in four Irish hospitals. Irish Pharm J 85(2):68-73. Available at: http://www.imsn.ie/Study\%20of\%20Medication\%20Safety.pdf

15. Kirke C, Irish Medication Safety Network (2009) Medication safety in hospitals. Ir Med J 102(10):339-341. PMID: 20108808. Available at: http://www.imj.ie//ViewArticleDetails.aspx?ContentID=3921

16. World Health Organization Anatomical Therapeutic Chemical (ATC) classification system. Available at: http://www.whocc.no/atc_ddd_index/

17. National Coordinating Council for Medication Error Reporting and Prevention (NCC MERP). Available at: http://www.nccmerp.org/

18. National Coordinating Council for Medication Error Reporting and Prevention (NCC MERP). What is a medication error? Available at: http://www.nccmerp.org/aboutMedErrors.html

19. World Health Organization (2009) More than words: Conceptual framework for the international classification on patient safety. Final technical report (v. 1.1). WHO: Geneva. Available at: http://www.who.int/patientsafety/taxonomy/icps_full_report.pdf

20. Hicks RW, Santell JP, Cousins DD, Williams RL (2004) MedMARx $5^{\text {th }}$ anniversary data report: A chartbook of 2003 findings and trends 1999-2003. USP Center for the Advancement of Patient Safety: Rockville, MD. Available at: http://psnet.ahrq.gov/resource.aspx?resourcelD $=1330$

21. Cohen M, Smetzer J, Tuohy N, Kilo C (2006) High-alert medications: safeguarding against errors. In: Cohen M (ed) Medication errors, 2nd edn. American Pharmaceutical Association, Washington DC, pp 317-412 